\title{
Ambipolar Electric Field and Potential in the Solar Wind Estimated from Electron Velocity Distribution Functions
}

\author{
Laura Berčič ${ }^{1}$ (D), Milan Maksimović ${ }^{2}$ (iD, Jasper S. Halekas ${ }^{3}$ (D), Simone Landi ${ }^{4,5}$ (D), Christopher J. Owen ${ }^{1}$ (D), \\ Daniel Verscharen $^{1,6}$ (1) , Davin Larson ${ }^{7}$ (i), Phyllis Whittlesey ${ }^{7}$ (i), Samuel T. Badman ${ }^{7}$ (i), Stuart. D. Bale ${ }^{7,8}$ (1) , Anthony W. Case ${ }^{9}$ (i)

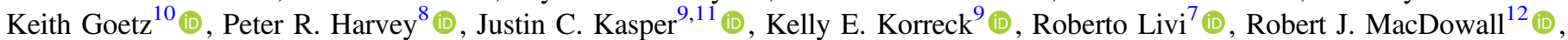 \\ David M. Malaspina ${ }^{13}$ (1D) Marc Pulupa $^{8}$ (i), and Michael L. Stevens ${ }^{9}$ (D) \\ ${ }^{1}$ Mullard Space Science Laboratory, University College London, Dorking, RH5 6NT, UK; 1.bercic@ucl.ac.uk \\ ${ }^{2}$ LESIA, Observatoire de Paris, Université PSL, CNRS, Sorbonne Université, Unversité de Paris, 5 place Jules Janssen, F-92195 Meudon, France \\ ${ }^{3}$ Department of Physics and Astronomy, University of Iowa, Iowa City, IA 52242, USA \\ ${ }^{4}$ Physics and Astronomy Department, University of Florence, Sesto Fiorentino, Italy \\ ${ }^{5}$ INAF-Osservatorio Astrofisico di Arcetri, Firenze, Italy \\ ${ }^{6}$ Space Science Center, University of New Hampshire, 8 College Road, Durham, NH 03824, USA \\ ${ }^{7}$ Physics Department, University of California, Berkeley, CA, USA \\ ${ }^{8}$ Space Sciences Laboratory, University of California, Berkeley, CA 94720-7450, USA \\ ${ }^{10}$ School of Physics and Astronomy, University of Minnesota, Minneapolis, MN 55455, USA \\ ${ }^{11}$ University of Michigan, Ann Arbor, MI, USA
${ }^{12}$ Solar System Exploration Division, NASA/Goddard Space Flight Center, Greenbelt, MD 20771, USA \\ ${ }^{13}$ Astrophysical and Planetary Sciences Department, University of Colorado, Boulder, CO, USA \\ Received 2021 June 9; revised 2021 August 12; accepted 2021 August 17; published 2021 November 3
}

\begin{abstract}
The solar wind escapes from the solar corona and is accelerated, over a short distance, to its terminal velocity. The energy balance associated with this acceleration remains poorly understood. To quantify the global electrostatic contribution to the solar wind dynamics, we empirically estimate the ambipolar electric field $\left(E_{\|}\right)$and potential $\left(\Phi_{\mathrm{r}, \infty}\right)$. We analyze electron velocity distribution functions (VDFs) measured in the near-Sun solar wind between $20.3 R_{S}$ and $85.3 R_{S}$ by the Parker Solar Probe. We test the predictions of two different solar wind models. Close to the Sun, the VDFs exhibit a suprathermal electron deficit in the sunward, magnetic-field-aligned part of phase space. We argue that the sunward deficit is a remnant of the electron cutoff predicted by collisionless exospheric models. This cutoff energy is directly linked to $\Phi_{\mathrm{r}, \infty}$. Competing effects of $E_{\|}$and Coulomb collisions in the solar wind are addressed by the Steady Electron Runaway Model (SERM). In this model, electron phase space is separated into collisionally overdamped and underdamped regions. We assume that this boundary velocity at small pitch angles coincides with the strahl break-point energy, which allows us to calculate $E_{\|}$. The obtained $\Phi_{\mathrm{r}, \infty}$ and $E_{\|}$agree well with theoretical expectations. They decrease with radial distance as power-law functions with indices $\alpha_{\Phi}=-0.66$ and $\alpha_{\mathrm{E}}=-1.69$. We finally estimate the velocity gained by protons from electrostatic acceleration, which equals $77 \%$ calculated from the exospheric models, and 44\% from the SERM model.
\end{abstract}

Unified Astronomy Thesaurus concepts: Solar wind (1534); Space plasmas (1544); Interplanetary particle acceleration (826); Collision processes (2065); Space vehicle instruments (1548)

\section{Introduction}

The solar wind is a continuous outflow of plasma from the hot solar corona (Parker 1958). The particles escaping the Sun are mostly electrons and protons, with a smaller population of heavier ions. Over a small radial distance, these particles reach bulk velocities of order a few $100 \mathrm{~km} \mathrm{~s}^{-1}$. The nature of the acceleration mechanisms converting the coronal thermal energy to the solar wind kinetic energy remains one of the most important open questions in heliophysics.

The terminal velocity of the solar wind is closely related to the density and temperature of the solar coronal plasma. These can be estimated remotely through spectroscopy and multifrequency radio imaging (Mercier \& Chambe 2015). In coronal holes, which are regions of open magnetic field lines along which plasma can flow freely in the radial direction, the typical electron temperature is 0.79 MK (David et al. 1998; Cranmer 2002), while much higher temperatures are found on the edges of coronal holes and in active regions (Stansby et al. 2021).
In coronal holes, the proton distributions appear hotter than those of electrons, and anisotropic with $T_{\perp}>T_{\|}$at radial distance $\sim 3 R_{S}$ (Cranmer 2002). Heavier ion distributions are strongly anisotropic at these distances with $T_{\perp} / T_{\|}$ranging between 10 and 100 (Kohl et al. 1998). Preferential perpendicular ion heating is believed to contribute to the solar wind acceleration (Munro \& Jackson 1977) through mechanisms like stochastic heating (Chandran et al. 2010; Bourouaine \& Chandran 2013), ion-cyclotron resonance (Dusenbery \& Hollweg 1981; Hollweg 1999; Li et al. 1999; Marsch \& Tu 2001; Tu \& Marsch 2001), or the dissipation of turbulence (Bieber et al. 1996; Oughton et al. 2001; Verdini et al. 2010; Karimabadi et al. 2013; Matthaeus et al. 2015; Agudelo Rueda et al. 2021).

In the case of electrons, a non-Maxwellian coronal velocity distribution function (VDF) with an excess of high-energy electrons can alone accelerate the solar wind protons to velocities above $700 \mathrm{~km} \mathrm{~s}^{-1}$ (Maksimovic et al. 1997; Zouganelis et al. 2004). The radial evolution of the collisionless, expanding solar wind is captured by the exospheric solar 
wind models (Jockers 1970; Lemaire \& Scherer 1970, 1971; Pierrard et al. 1999; Maksimovic et al. 2001; Zouganelis et al. 2005). The drivers of the solar wind in these models are the solar wind electrons. Due to their small mass, their thermal velocity just above the solar surface is large enough for the majority of electrons to escape the Sun's gravity. However, this is not the case for the heavier protons. A global electric polarization field, also referred to as the ambipolar electric field $\left(E_{\|}\right)$, builds up, accelerating the protons and decelerating the electrons. It preserves equal ambipolar diffusion of ions and electrons in the radial direction. This study focuses on the quantification of $E_{\|}$in the solar wind and thus its contribution to the total solar wind acceleration.

$E_{\|}$decreases with radial distance from the Sun and has a magnitude of order a few $\mathrm{nV} \mathrm{m}^{-1}$ in the inner heliosphere (e.g., Berčič et al. 2021), thus it is practically undetectable by spacecraft electric field antennas. However, electron VDFs measured in the near-Sun solar wind are highly affected by $E_{\|}$ and thus can tell us something about its properties.

Electron VDFs in the solar wind have a complex structure and are commonly modeled with three components. Lowenergy electrons belong to the almost isotropic core population and are well represented by a Maxwellian distribution. Higher energy electrons belong to either the isotropic halo population or the magnetic-field-aligned, beam-like population called the strahl (Feldman et al. 1975; Schwartz \& Marsch 1983; Pilipp et al. 1987; Maksimovic et al. 1997, 2005; Štverák et al. 2008, 2009; Tao et al. 2016). Another electron feature is often observed in the near-Sun solar wind-a relative deficit of electrons compared to the Maxwellian core model appears in the suprathermal energy range in the portion of phase space, opposite to the strahl direction (Halekas et al. 2020, 2021a; Berčič et al. 2020, 2021; Bercic et al. 2021). The statistical properties of this sunward deficit are presented by Halekas et al. (2021b).

\subsection{The Exospheric Prediction}

A deficit of sunward moving electrons is also a feature of collisionless exospheric models, where it is referred to as the "electron cutoff." The electron VDF at any radial distance in these models is separated into two parts: anti-sunward-moving electrons with energy greater than the ambipolar electric potential energy $\left(\mathcal{E}_{\Phi}\right)$ represent the escaping electrons, which focus toward the magnetic field direction and form the strahl population; ballistic electrons with energies less than $\mathcal{E}_{\Phi}$ represent the core population. In the sunward direction, these electrons are limited by the cutoff energy $\left(E_{\mathrm{C}}\right)$ corresponding to the ambipolar potential between their location and the asymptotic value at large heliocentric distances, where $r \rightarrow \infty$ (Jockers 1970; Maksimovic et al. 2001):

$$
\Phi_{\mathrm{r}, \infty}=E_{\mathrm{C}} / e
$$

where $e$ is the electron charge and $E_{\mathrm{C}}$ is defined in the Sun's rest frame. In this paper, we identify $E_{\mathrm{C}}$ related to the sunward deficit in the electron VDFs observed by the Parker Solar Probe (PSP) and use the exospheric prediction to estimate $\Phi_{\mathrm{r}, \infty}$.

\subsection{The Electron Runaway Model Prediction}

A different theoretical description of the solar wind electrons is proposed by Scudder (1996, 2019a, 2019b, 2019c) called the Steady Electron Runaway Model (SERM). SERM accounts for the behavior of weakly collisional electrons in the large-scale ambipolar electric field. Instead of assuming local thermodynamic equilibrium, it proposes a steady electron runaway effect. The Dreicer electric field $\left(E_{\mathrm{D}}\right)$ (Dreicer 1959,1960$)$ is used to compare the strength of $E_{\|}$to the collisionality of the system. $E_{\mathrm{D}}$ is defined as the constant electric field strength needed to accelerate a thermal particle to twice its velocity in one collision time (Dreicer 1959, 1960):

$$
E_{\mathrm{D}}=\frac{2 k_{\mathrm{B}} T_{\mathrm{c} \|}}{e \lambda_{\mathrm{mfp}}},
$$

where $k_{\mathrm{B}}$ is the Boltzmann constant, $T_{\mathrm{c} \|}$ is the core electron temperature parallel to the magnetic field and $\lambda_{\mathrm{mfp}}$ is the collisional electron mean free path.

The resulting electron VDFs consist of collisionally overdamped and underdamped regions, separated by a 2D separatrix in phase space (Fuchs et al. 1986). The overdamped region corresponds to the core population and the underdamped region to the suprathermal populations. The boundary for small pitch angles can be related to the energy at which the core transitions to the strahl, the strahl break-point energy $\left(E_{\mathrm{BP}}\right)$, which we also identify in the PSP electron VDFs. $E_{\|}$then follows from (Scudder 2019a)

$$
E_{\|}=\frac{\alpha k_{\mathrm{B}} T_{\mathrm{c} \|}}{E_{\mathrm{BP}}} E_{\mathrm{D}},
$$

where $\alpha=3$ (Dreicer 1960) and $E_{\mathrm{BP}}$ is defined in the plasma frame.

In Section 2 we describe the data set and the method we use to obtain $\Phi_{\mathrm{r}, \infty}$ and $E_{\|}$. Section 3 presents our results, which we discuss in Section 4. We summarize our findings and draw conclusions in Section 5.

\section{Data Analysis}

\subsection{Data Set}

This work is based on the analysis of the electron VDFs measured by PSP (Fox et al. 2016), a heliospheric mission exploring the young solar wind near the Sun. The SPAN Electron (SPAN-E) instrument (Whittlesey et al. 2020), part of the SWEAP investigation package on PSP (Kasper et al. 2016), measures the solar wind electrons. SPAN-E consists of two toroidal top-hat analyzers, SPAN-A and SPAN-B, which together cover almost a full-sky field of view (FOV). A small portion of the combined FOV in the direction of the Sun is blocked by the spacecraft's heat shield, which protects the payload from direct solar radiation. During encounter periods this FOV obstruction affects measurements taken within $\sim 10^{\circ}$ from the radial direction. Each of the top-hat analyzers measures electron velocity directions with 8 small $\left(6^{\circ}\right)$ and 8 large $\left(24^{\circ}\right)$ azimuth anodes, and 16 elevation deflection states, which vary in angular width from $10^{\circ}$ to $15^{\circ}$. The electron energy is sampled in 32 log-spaced bins covering the energy range between $2 \mathrm{eV}$ and $2 \mathrm{keV}$ with a $\Delta E / E$ of $7 \%$. The duration of a full 3D sweep over all energy and deflection bins is $0.218 \mathrm{~s}$.

We use electron VDFs collected in Survey Mode during encounter periods, which consist of multiple full 3D sweeps integrated over time. The presented data was collected during PSP's perihelion passages 4 (2020 January 23-February 3) and 5 (2020 May 30-June 15), with the closest approach at $27.8 R_{S}$, and 6 (2020 September 16-October 5) and 7 (2021 January 


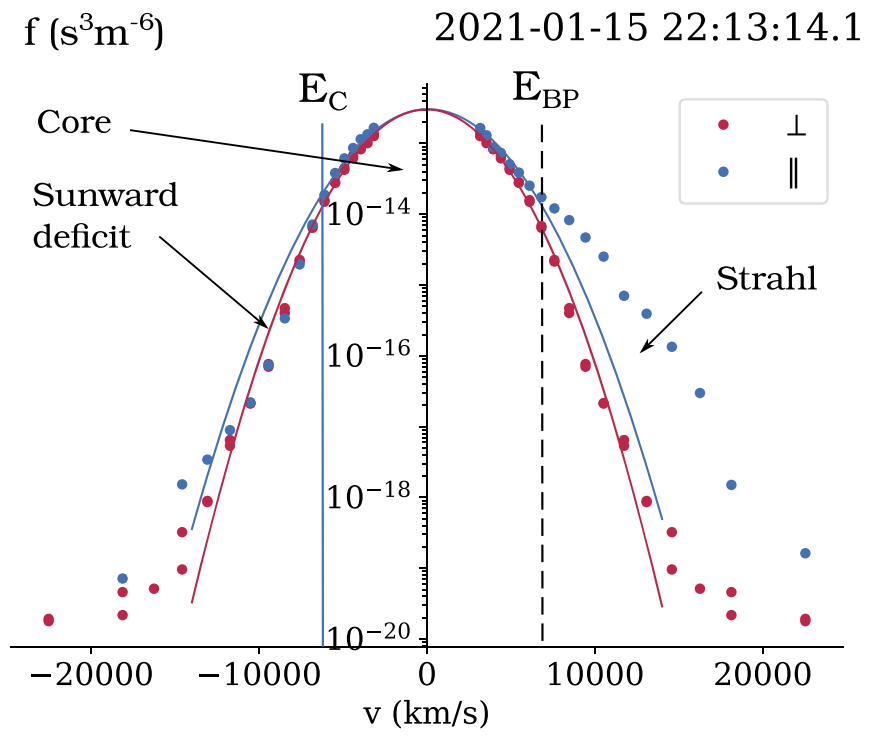

Figure 1. Parallel and perpendicular cuts through an electron VDF in the magnetic-field-aligned, instrument-centered frame. Blue and red dots are the measured points, while blue and red lines denote the parallel and perpendicular cuts through the 3D bi-Maxwellian fit to the core electron population. The blue and black dashed lines mark the transitions between the suprathermal deficit and the core $\left(E_{\mathrm{C}}\right)$, and between the strahl and the core $\left(E_{\mathrm{BP}}\right)$ in the parallel direction.

10-28), with the closest approach at $20.3 R_{S}$. We thus investigate the region between $20.3 R_{S}(0.10 \mathrm{au})$ and $85.3 R_{S}$ $(0.40 \mathrm{au})$. For encounters 4,5 , and 7 , the integration time is set to $13.95 \mathrm{~s}$, and for encounter 6 it is set to $3.49 \mathrm{~s}$. Detailed descriptions of the SPAN-E instruments and their operating modes are provided by Whittlesey et al. (2020).

In our analysis we also use the magnetic field vector measured by the triaxial fluxgate magnetometer (MAG) part of the FIELDS investigation suite (Bale et al. 2016) and the proton velocity moment derived from the proton VDFs detected by the SPAN Ion (SPAN-I) instrument (Kasper et al. 2016). Both magnetic field and proton velocity are available with higher or equal cadence than the electron VDFs and are thus averaged over the SPAN-E integration periods to match the electron data.

Our main reason for using the data from four orbits of PSP, out of eight in total to date, is the availability of the SPAN-I data, which improve significantly after encounter 3. A Faraday cup instrument (SPC; Case et al. 2019) also measures solar wind proton velocity on PSP but discrepancies between the two instruments exist (Woodham et al. 2021). We choose to use data from SPAN-I, which provides more accurate data closer to the Sun, where the aberration allows the solar wind protons to fly into the instrument protected by the heat shield. Other reasons for the data selection are the changes made in the integration time and elevation deflection tables over the course of the first three orbits, ensuring optimal operation of SPAN-E during the later encounters. We also exclude the electron VDFs obtained at larger distances from the Sun during the PSP cruise phase, which are integrated over larger time intervals. Our goal is to obtain a consistent data set of electron VDFs and use it to investigate features typical for the near-Sun environment.

\subsection{Method}

Following the example of previous studies (e.g., Berčič et al. 2019, 2020; Halekas et al. 2020) we analyze electron VDFs in the magnetic-field-aligned, plasma rest frame. We rotate the VDFs given in the SPAN-A and SPAN-B instrument frames using the magnetic field vector and shift them using the spacecraft and the solar wind velocities. We show an example of an observed VDF in Figure 1 as cuts along and perpendicular to the magnetic field direction. We already noted the features investigated in this work along the parallel direction: the suprathermal deficit and the strahl. Our aim is to identify the energies at which the electron VDF starts to depart from the Maxwellian core in the directions parallel and anti-parallel to the magnetic field.

We fit the core with a bi-Maxwellian distribution function:

$$
\begin{aligned}
f_{\mathrm{c}}\left(v_{\perp 1}, v_{\perp 2}, v_{\|}\right)= & A_{\mathrm{c}} \exp \left(-\frac{\left(v_{\perp 1}-v_{\mathrm{c} \perp 1}\right)^{2}}{w_{\mathrm{c} \perp}^{2}}\right. \\
& \left.-\frac{\left(v_{\perp 2}-v_{\mathrm{c} \perp 2}\right)^{2}}{w_{\mathrm{c} \perp}^{2}}-\frac{\left(v_{\|}-v_{\mathrm{c} \|}\right)^{2}}{w_{\mathrm{c} \|}^{2}}\right),
\end{aligned}
$$

where $A_{\mathrm{c}}$ is the normalization factor, $w_{\mathrm{c} \perp}$ is the perpendicular core thermal velocity, $w_{\mathrm{c} \|}$ is the parallel core thermal velocity, $v_{\mathrm{c} \perp 1,2}$ are the two core perpendicular drift velocities, and $v_{\mathrm{c} \|}$ is the core parallel drift velocity. These quantities are our fit parameters, from which we obtain the core density as

$$
n_{\mathrm{c}}=A_{\mathrm{c}} \pi^{3 / 2} w_{\mathrm{c} \perp}^{2} w_{\mathrm{c} \|}
$$

and the core parallel and perpendicular temperatures as

$$
T_{\mathrm{c} \|}=\frac{m_{e} w_{\mathrm{c} \|}^{2}}{2 k_{\mathrm{B}}} \quad \text { and } \quad T_{\mathrm{c} \perp}=\frac{m_{e} w_{\mathrm{c} \perp}^{2}}{2 k_{\mathrm{B}}},
$$

where $m_{e}$ is the mass of an electron.

Even though $v_{\mathrm{c} \perp 1,2}$ are expected to be 0 (Pilipp et al. 1987, e.g.,), we allow for perpendicular core drifts to correct for possible errors in the measured solar wind velocity vector. The values of $v_{\mathrm{c} \perp 1,2}$ we obtain are small, and the resulting fit parameters are not strongly affected by it.

For the core fit, we only use the data points belonging to the core population, which we determine according to the electron energy and pitch angle. First, we avoid the inclusion of photoelectrons and secondary electrons reflected from the spacecraft by setting a lower energy limit to $35.7 \mathrm{eV} .^{14}$

Then, we avoid the inclusion of the strahl population through a two-step fitting procedure, which is based on the expected strahl break-point energy $\left(E_{\mathrm{BP}}\right)$ following from the kinetic solar wind model Binary Collisions in Plasmas (BiCoP; Berčič et al. 2021). We use this technique because we find that the core fit along the parallel direction is very sensitive to the selection of data points at small pitch angles. Assuming that $E_{\mathrm{BP}}$ coincides with the separatrix between the collisionally overdamped and underdamped regions (Scudder 2019a), $E_{\mathrm{BP}}$ is related to $T_{\mathrm{c} \|}$ as

$$
E_{\mathrm{BP}}=\frac{E_{\mathrm{D}}}{E_{\|}} 3 k_{\mathrm{B}} T_{\mathrm{c} \|}
$$

We perform the first fit to all measurements with pitch angles greater than $60^{\circ}$ and energies less than $132 \mathrm{eV}$. The parameters related to the first fit are marked with a tilde. $\widetilde{T}_{\mathrm{c}, \perp}$ obtained from this first fit is already very accurate as the strahl is narrow near the Sun and mainly affects the core electron fit along the

\footnotetext{
14 The same method is used by Berčič et al. (2020); however, Halekas et al (2020) fit and subtract the secondary electron peak. Both methods produce similar results.
} 


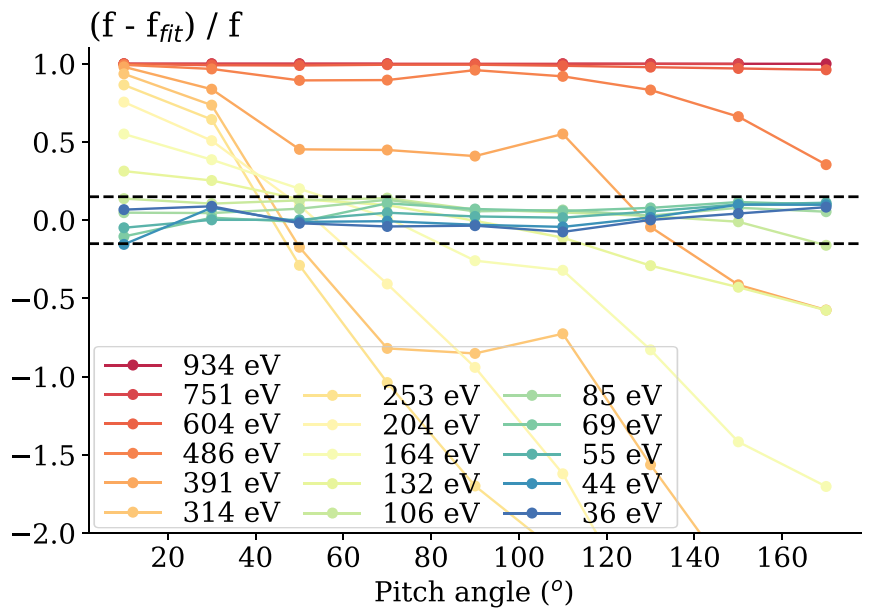

Figure 2. The normalized difference between the measured VDF and the core fit $\left(\Delta f_{\text {data,fit }}\right)$ binned in $20^{\circ}$ pitch-angle bins. Different colors mark separate instrument energy bins. The black dashed line shows the criteria used in the determination of $E_{\mathrm{C}}$ and $E_{\mathrm{BP}}$.

parallel direction. To obtain a zero-order estimation of $\widetilde{E}_{\mathrm{BP}}$, we assume $E_{\|} \approx E_{\mathrm{D}}$ and $\widetilde{T}_{\mathrm{c}, \perp} \approx \widetilde{T}_{\mathrm{c} \|}$, and calculate $\widetilde{E}_{\mathrm{BP}}$ as $\widetilde{E}_{\mathrm{BP}}=3 k_{\mathrm{B}} \widetilde{T}_{\mathrm{c} \perp}$. This energy is then used for the second (final) fit as an upper energy limit for the data points with pitch angles less than $60^{\circ}$.

To define the boundaries between the deficit and the core, and between the core and the strahl, we look for departures of the measured VDF from the fitted bi-Maxwellian core distribution. We calculate the normalized difference between the two in each measured point as

$$
\Delta f_{\text {data,fit }}=\frac{f_{\text {data }}-f_{\text {fit }}}{f_{\text {data }}},
$$

where $f_{\text {data }}$ are the measured values and $f_{\text {fit }}$ are the core fit values corresponding to the centers of the measurement bins in phase space. We bin $\Delta f_{\text {data,fit }}$ into $20^{\circ}$ wide pitch-angle bins and calculate the median value in each bin $\left(\operatorname{med}\left\{\Delta f_{\text {data, fit }}\right\}\right)$. This way, we avoid the possible effects of FOV blockage by the heat shield, which is $\sim 10^{\circ}$ wide in pitch angle, when the magnetic field is aligned with the radial direction (Kasper et al. 2016). Figure 2 shows these values for separate instrument energy bins on the example VDF from Figure 1. The value of $\Delta f_{\text {data,fit }}$ at low energies, represented by the blue part of the color-scale spectra, remains around 0 , which means that the biMaxwellian fit represents well the electron VDF in this energy range. High energies are plotted in red and reach 1, which indicates that the measured VDF is much greater than the obtained core fit. The departure from the core fit at high energies is expected due to the presence of the halo population. For the energies in between we observe a pitch-angle dependent evolution of the departures from the bi-Maxwellian core.

We define the strahl break-point energy $\left(E_{\mathrm{BP}}\right)$ as the lowest energy at which med $\left\{\Delta f_{\text {data,fit }}\right\}$ exceeds the value of 0.15 in the $0^{\circ}-20^{\circ}$ pitch-angle bin. If this energy is greater than $40 \mathrm{eV}$ and less than $700 \mathrm{eV}$, we consider it a successful determination of $E_{\mathrm{BP}}$.

We define the electron cutoff energy $\left(E_{\mathrm{C}}\right)$ as the lowest energy at which med $\left\{\Delta f_{\text {data,fit }}\right\}$ decreases below the value of
-0.15 in the $160^{\circ}-180^{\circ}$ pitch-angle bin. Black dashed lines mark the limit values in Figure 2. The cutoff is only considered for instances when $\operatorname{med}\left\{\Delta f_{\text {data,fit }}\right\}$ at any energy is less than -0.5 . We move $E_{\mathrm{C}}$ to the Sun rest frame using the solar wind velocity. The method is considered successful if the identified energy lies within an interval from 60 to $400 \mathrm{eV}$.

We calculate $\lambda_{\mathrm{mfp}}$ used in Equation (2) from the ratio between the electron parallel core thermal velocity $\left(w_{\mathrm{c} \|}\right)$ and the electron collision frequency, which we obtain from the relation between the electron density and temperature (e.g., Salem et al. 2003)

$$
\nu_{\mathrm{e}}=2.9 \times 10^{-6} n_{\mathrm{c}} T_{\mathrm{c} \|}^{-3 / 2} \ln \Lambda
$$

where $\ln \Lambda$ is the Coulomb logarithm defined as

$$
\ln \Lambda=\ln \left(\frac{12 \pi\left(\epsilon_{0} k_{\mathrm{B}} T_{\mathrm{c} \|}\right)^{3 / 2}}{n_{\mathrm{c}}^{1 / 2} e^{3}}\right),
$$

and $\epsilon_{0}$ is the vacuum permittivity.

\section{Results}

The Figure 3 shows the same electron VDF as shown in Figure 1 but plotted against $v_{\|}$and $v_{\perp}$. We show a single VDF using four different 2D representations, which we obtain by the integration along the angle perpendicular to the magnetic field. All plots show the distribution in the magnetic-field-aligned frame centered on the core parallel velocity. The representation marked original shows the measured VDF values with a logarithmic color scale. In the representation marked scaled, each energy bin-i.e., each circular belt in $\left(v_{\|}, v_{\perp}\right)$ parameter space-is scaled to a value between 0 and 1 , where 1 corresponds to the maximum value of the VDF in the given energy belt. This representation removes the information about the absolute value of the VDF and its strong gradient in energy. The benefit of the scaled VDF is the exposure of smaller anisotropic features at all energies. In cases for which two features arise in the same energy bin, the scaled VDFs can be misleading, though, as they highlight only the stronger feature. The representation marked normalized is obtained by dividing each of the VDF values with $f\left(v_{\perp}, v_{\|}=0\right)$ of the associated energy bin. Pitch-angle directions in which the distribution function is less than $f\left(v_{\perp}, v_{\|}=0\right)$, appear in green, and those in which the distribution function is greater than $f\left(v_{\perp}, v_{\|}=0\right)$ appear in red. The representation marked fit-normalized shows the logarithm of electron VDF divided by the core fit. Yellow phase space regions are well represented by a bi-Maxwellian distribution while departures are seen in red and blue colors.

We use these representations to obtain a better understanding of the 2D shape of the features in the electron VDF. Electrons at energies below $\sim 100 \mathrm{eV}$ are predominantly members of the almost isotropic core population, which shows almost no pitchangle variation in all representations. At positive $v_{\|}$, we observe a distinct strahl, the shape of which is most clearly defined in the scaled VDF. Its width in terms of perpendicular velocity appears almost constant, which gives a decreasing angular pitch-angle width with increasing electron energy. The sunward deficit is present at negative $v_{\|}$, and shows in green and blue colors in the fit-normalized VDF. This feature not only persists at high pitch angles (close to $180^{\circ}$ ), but it forms a circular belt in phase space at some energies reaching to the strahl population at small pitch angles. The absence of the deficit in 

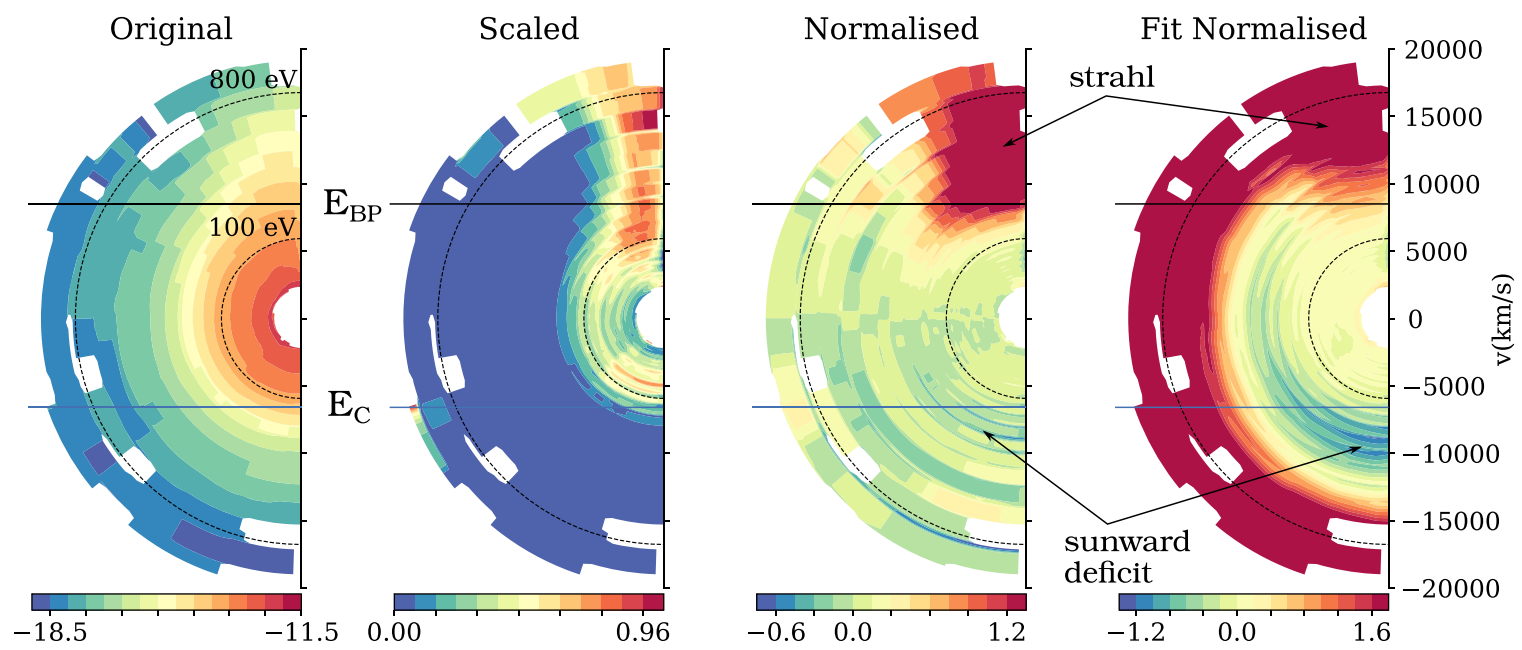

Figure 3. The same electron VDF as shown in Figures 1 and 3, plotted as a function of parallel and perpendicular velocity. The VDF is shown in the magnetic field aligned frame, centered on the core parallel velocity. Plots from left to right present: the original VDF; the scaled VDF, where values in each energy bin are scaled between 0 and 1 ; the normalized VDF, where the original VDF is divided by the perpendicular VDF cut $\left(f\left(v_{\perp}, v_{\|}=0\right)\right)$; and the $f i t$-normalized $\mathrm{VDF}$, where the $\mathrm{VDF}$ is divided by the core electron fit.

the normalized VDF tells us that this feature is close to isotropic, existing also at pitch angles around $90^{\circ}$.

In total, we successfully fit 510,610 full 3D electron VDFs, out of which we determine $E_{\mathrm{BP}}$ in $98.3 \%$ and $E_{\mathrm{C}}$ in $55.4 \%$ of the cases. We statistically visualize the energy at which these two boundaries occur in a histogram in Figure 4, where the bin sizes correspond to the instrument's energy resolution. $E_{\mathrm{BP}}$ and $E_{\mathrm{C}}$ are strongly correlated and the mean ratio between the two $\left(E_{\mathrm{C}} / E_{\mathrm{BP}}\right)$ equals to 1.42 .

We plot $\Phi_{\mathrm{r}, \infty}$ obtained from $E_{\mathrm{C}}$ through Equation (1) on a 2D histogram against radial distance in Figure 5(a). The red dots mark the median values, and the corresponding error bars show the intervals of one standard deviation of the data set in each radial bin. The potential $\Phi_{\mathrm{r}, \infty}$ decreases with radial distance and takes the values between 300 and $60 \mathrm{~V}$. The step histograms above the plot compare the number of available data points in each radial bin to the number of data points for which $E_{\mathrm{C}}$ is found. According to these histograms, the proportion of the electron VDFs with a sunward deficit decreases with increasing radial distance. form

We fit all data points with a power-law distribution of the

$$
\Phi_{\mathrm{r}, \infty}=\Phi_{0} r^{\alpha_{\Phi}},
$$

where $\Phi_{0}$ is a constant and $\alpha_{\Phi}$ is the power-law index. The best fit is plotted with the black dashed line in Figure 5(a) and the fitting parameters noted in the legend. The black dotted line shows a fit with $\alpha_{\Phi}$ fixed to the value obtained from the radial evolution of the ambipolar potential in BiCoP simulations (Berčič et al. 2021).

We show a similar plot, but for $E_{\|}$calculated from $E_{\mathrm{BP}}$ through Equation (3) in Figure 5(b). The absolute values of $E_{\|}$ span between 0.5 and $10 \mathrm{nV} \mathrm{m}^{-1}$ and decrease faster with radial distance than $\Phi_{\mathrm{r}, \infty}$. We fit the data points with a power law

$$
E_{\|}=E_{0} r^{\alpha_{\mathrm{E}}}
$$

and mark the values of the fitting parameters $E_{0}$ and $\alpha_{\mathrm{E}}$ in the legend. As for the case of the ambipolar potential, we show a

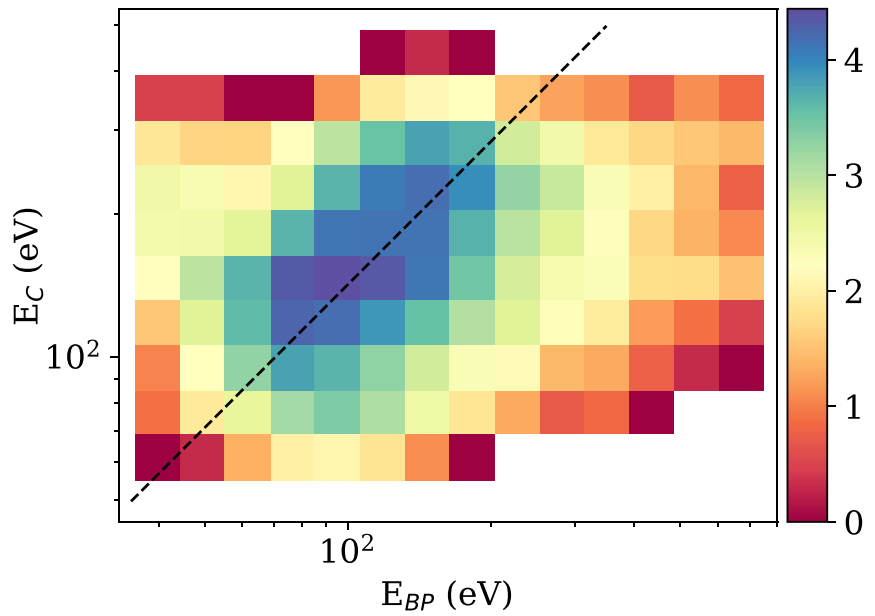

Figure 4. A $2 \mathrm{D}$ histogram showing the relation between $E_{\mathrm{BP}}$ and $E_{\mathrm{C}}$. The bin size corresponds to the energy resolution of the SPAN-E instrument. The color scale represents the logarithm of the number of instances in each bin $(\log (\#))$. The dashed line denotes $E_{\mathrm{C}}=1.42 E_{\mathrm{BP}}$.

second fit as a dotted line, representing a power-law function with an index equal to the one obtained from the $\mathrm{BiCoP}$ model.

In the bottom row of Figure 5, we explore how the boundary energies $E_{\mathrm{C}}$ and $E_{\mathrm{BP}}$ compare to $T_{\mathrm{c} \|}$ for different radial distances. The ratio $E_{\mathrm{C}} / T_{\mathrm{c} \|}$ slightly decreases with radial distance, spanning from a mean value of 5.7 close to the Sun, to 3.3 farther away. The ratio $E_{\mathrm{BP}} / T_{\mathrm{c} \|}$ exhibits an opposite trend, increasing from the mean value of 3.0-7.8.

\section{Discussion \\ 4.1. Ambipolar Electric Potential $\left(\Phi_{\mathrm{r}, \infty}\right)$}

The electron deficit in the suprathermal energy range is reported already by Pilipp et al. (1987), analyzing Helios data that cover distances down to $65 R_{S}$ from the Sun. In the PSP data, the sunward deficit is a common feature (Halekas et al. 2020; Berčič et al. 2020), which contributes significantly to the net electron heat-flux (Halekas et al. 2021a). The characteristics of the sunward deficit and their relation to different solar wind parameters are investigated by Halekas et al. (2021b). 
(a)
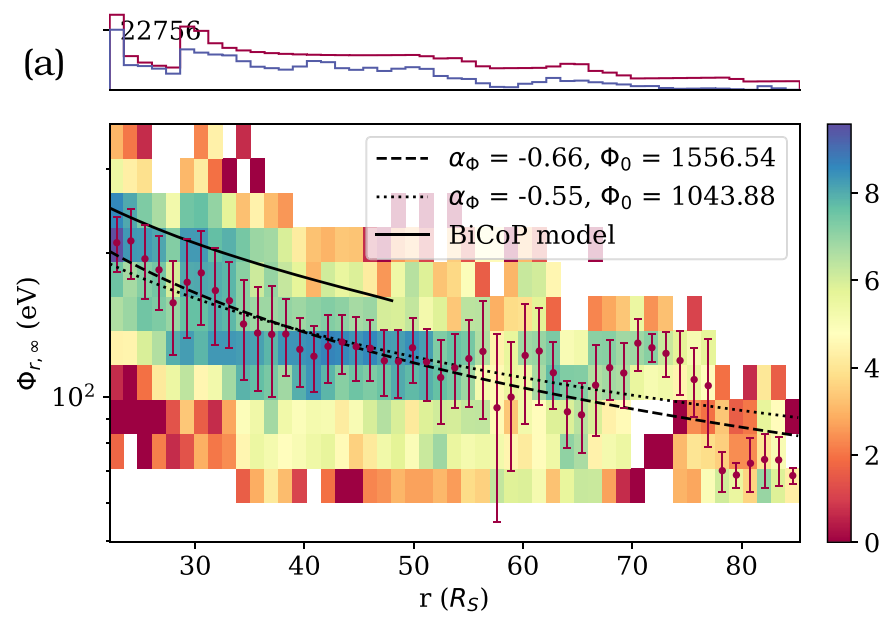

(c)

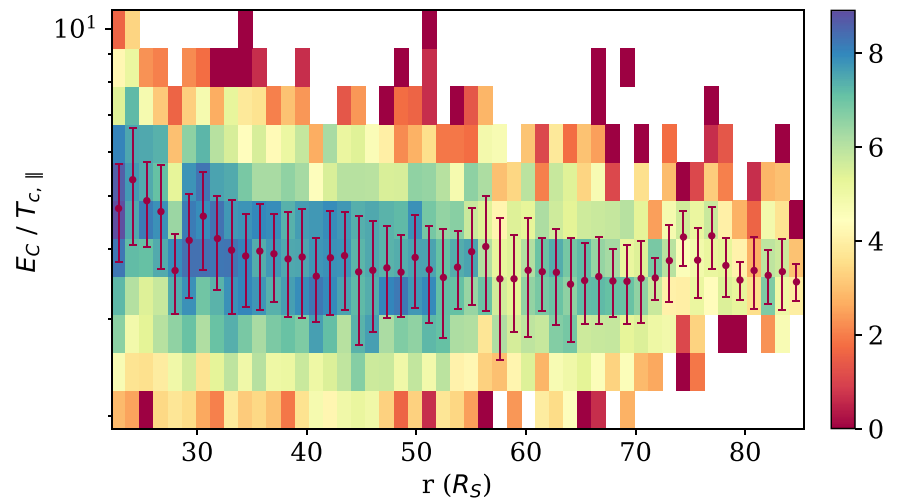

(b)
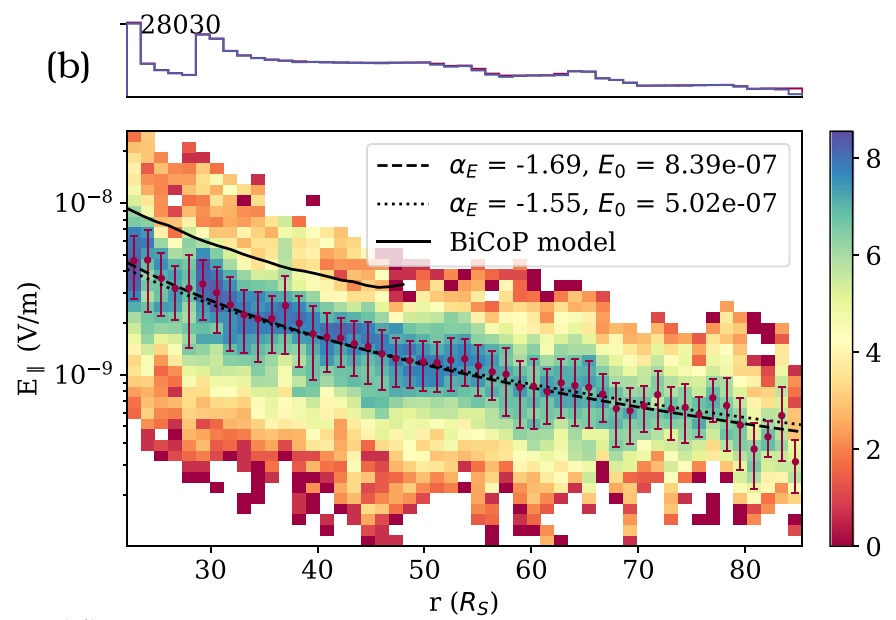

(d)

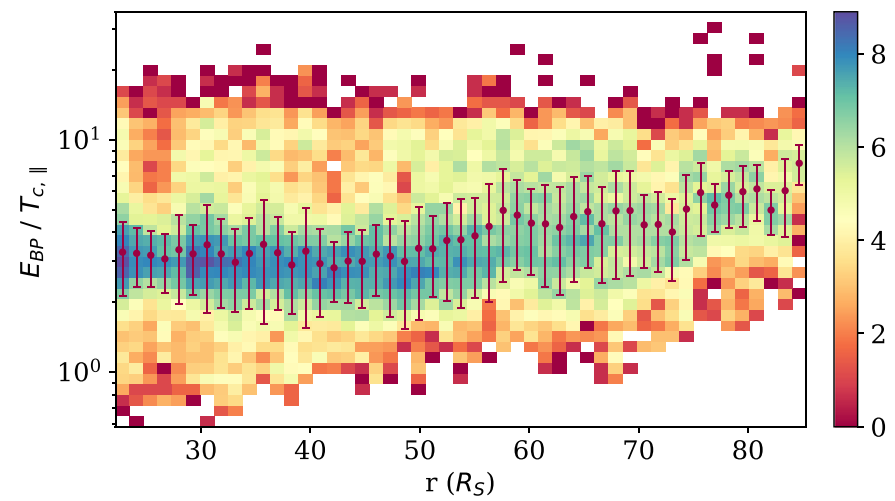

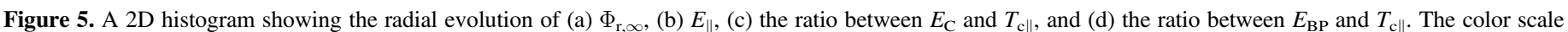

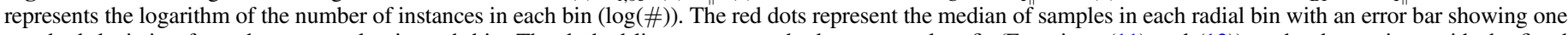

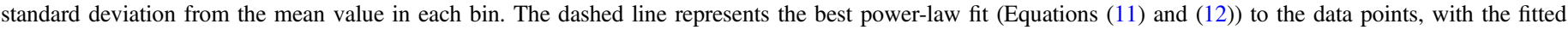

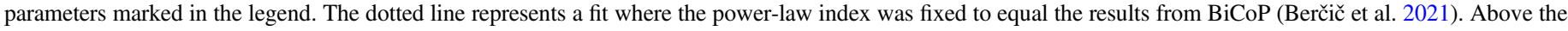

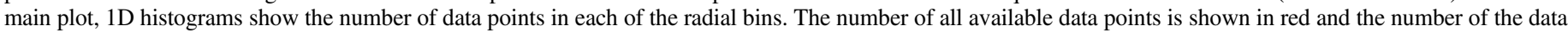
points used in the 2D histogram in blue.

In the collisionless exospheric models (Jockers 1970; Lemaire \& Scherer 1970, 1971; Maksimovic et al. 1997; Pierrard et al. 1999; Maksimovic et al. 2001; Zouganelis et al. 2004) no electrons with energies greater than the electric potential energy $\left(\mathcal{E}_{\Phi}\right)$ exist in the sunward portion of the electron VDF. Therefore, knowing the electron cutoff energy, we can obtain the value of the electric potential at any radial distance in the exosphere (Equation (1)). In kinetic models accounting additionally for Coulomb collisions, the electron cutoff is smoothed, appearing more like a deficit compared to the expected Maxwellian core VDF (Pierrard et al. 2001; Berčič et al. 2021). These models reproduce the observed radial profiles of the electron core properties, like density, temperature, and anisotropy. They also reproduce the strahl; however, they fail to produce the halo population. Electron VDFs observed close to the Sun (see example in Figures 1 and 3) exhibit only a tenuous halo population and are thus very similar to the VDFs from the $\mathrm{BiCoP}$ simulation (see Figures 7 and 8 by Berčič et al. 2021). Comparing the normalized VDF in Figure 3 with the normalized VDF in Figure 7 in Berčič et al. (2021), we see, however, that the shape of the sunward deficit is slightly different. In PSP data the deficit exists at pitch angles $\gtrsim 45^{\circ}$, while in BiCoP it only takes the angles $\gtrsim 135^{\circ}$.

In the near-Sun solar wind (at $\sim 34 R_{S}$ ), Coulomb collisions only effectively scatter the strahl electrons with energies smaller than $250 \mathrm{eV}$ (Horaites et al. 2018; Boldyrev \& Horaites 2019; Berčič et al. 2021). The scattering of the strahl at higher energies and the creation of the halo population are therefore attributed to other phenomena, including waveparticle interactions (Vocks et al. 2005; Kajdič et al. 2016; Verscharen et al. 2019; Jagarlamudi et al. 2020; Jeong et al. 2020; Cattell et al. 2021) and scattering by background turbulence (Pagel et al. 2007; Saito \& Gary 2007). Observational studies by Štverák et al. (2009) and Halekas et al. (2020) suggest that the halo is more prominent farther from the Sun, which could be the reason why the sunward deficit has not been observed at larger radial distances (Figure 5(a)).

A recent study by Bercic et al. (2021), who analyze Solar Orbiter (Müller et al. 2020) in situ measurements of solar wind electrons, shows that the sunward deficit can drive the growth of quasi-parallel whistler waves, leading to quasi-linear electron diffusion in velocity space. This newly proposed instability tends to fill the sunward deficit and may thus also be the reason why the electron cutoff ceases to exist at larger radial distances.

Another possibility for the disappearance of the deficit could simply be the Coulomb collisions: as $\Phi_{\mathrm{r}, \infty}$ decreases with radial distance it moves to the energy range where electron collisions are frequent. They could completely erase the residue of the cutoff. 
Ballistic electrons, in exospheric models representing the electron core population, are limited in energy to a range $\lesssim \mathcal{E}_{\Phi}$. Therefore, we expect that the core electron temperature $\left(T_{\mathrm{c}}\right)$ follows the radial evolution of $\Phi_{\mathrm{r}, \infty}$. This would show as a constant ratio between $E_{\mathrm{C}}$ and $T_{\mathrm{c} \|}$ in Figure 5(c). The observed variation in the ratio is not large, but a slight decreasing trend is present, mostly for radial distances below $35 R_{S}$. The increase in $E_{\mathrm{C}} / T_{\mathrm{c} \|}$ with decreasing heliocentric distance suggests the increasing importance of Coulomb collisions, which smear the exospheric VDF features and raise $E_{\mathrm{C}}$.

We compare the measured $\Phi_{\mathrm{r}, \infty}$ to the results of a kinetic numerical model (BiCoP) (Berčič et al. 2021), which builds up a supersonic radially expanding solar wind taking into account binary particle collisions and the self-consistent $E_{\|}$(Landi \& Pantellini 2001, 2003). The simulation box spans from 3 to $49 R_{S}$, thus overlapping with approximately half of the radial interval shown in this study. The self-consistently obtained $\Phi_{\mathrm{r}, \infty}$ from BiCoP is added to Figure 5(a) and evolves with radial distance as a power law with an index $\alpha_{\Phi, \mathrm{BCP}}=-0.55$. This result is close to the power-law index obtained in our observational study, $\alpha_{\Phi}=-0.66$. In Figure 5(a), we show a second fit to the data points with fixed $\alpha_{\Phi}=-0.55$ (dotted line) to emphasize that the power-law index is a sensitive fitting coefficient, and may vary across different solar wind streams. For the scope of this work, we only calculate the average properties of all of the solar wind measured during the four PSP encounters.

Our experimentally determined $\alpha_{\Phi}$ diverges from the powerlaw index in collisionless exospheric models, $\alpha_{\Phi, \text { Exo }}=-1.33$ (e.g., Meyer-Vernet \& Issautier 1998; Zouganelis et al. 2004), indicating that collisions play an important role in the radial evolution of $\Phi_{\mathrm{r}, \infty}$ and in the ambipolar solar wind acceleration. An analytical solution of the drift-kinetic equation including the effects of Coulomb collisions (Boldyrev et al. 2020) gives a power law with $\alpha_{\Phi, \mathrm{DK}}=-0.4$, which is closer to our observations.

We add $\Phi_{\mathrm{r}, \infty}$ obtained with a medium-collisional BiCoP run (MC in Berčič et al. 2021) to Figure 5(a). The modeled values are within the range of the observed values, even though the BiCoP boundary parameters-electron and proton temperature at $3 R_{S}$ set to $121 \mathrm{eV}$-differ from the expected coronal temperatures. Electrons in the corona are observed to be colder, $T_{\mathrm{e}} \sim 86 \mathrm{eV}$ (1 MK; Cranmer 2002; Berčič et al. 2020; Stansby et al. 2021), while the proton temperature is expected to be greater. This difference in temperature between the two species and the preferential perpendicular heating of solar wind protons potentially result in the observed $\Phi_{\mathrm{r}, \infty}$, even when the electron temperature at the origin is less than the electron temperature assumed in $\mathrm{BiCoP}$ simulations. Initializing $\mathrm{BiCoP}$ runs with different coronal temperatures for electrons and protons, and with varying proton anisotropies, would give further insight into this phenomenon.

\subsection{Ambipolar Electric Field $\left(\mathrm{E}_{\|}\right)$}

The SERM (Scudder 1996, 2019a, 2019b, 2019c) accounts for the effects of the global ambipolar electric field $\left(E_{\|}\right)$in the presence of Coulomb collisions. The Dreicer electric field $\left(E_{\mathrm{D}}\right.$, Equation (2)) serves as a measure of the importance of these two competing phenomena (Dreicer 1959, 1960). We use the measured $E_{\mathrm{BP}}$ to estimate the ambipolar electric field in the solar wind (Equation (3)). Before discussing the properties of the observed $E_{\|}$, we compare $E_{\mathrm{BP}}$ in the near-Sun solar wind to already existing studies.

The idea that the separatrix between the thermal and suprathermal electron populations contains information about the electron kinetics is discussed in early observational studies (Feldman et al. 1975; Pilipp et al. 1987). Scudder \& Olbert (1979) theoretically predict that the break-point energy scales with the local electron temperature as $E_{\mathrm{BP}}=7 k_{\mathrm{B}} T_{\mathrm{c}}$. This value agrees with the break-point between the core and the halo obtained by Štverák et al. (2009), who analyze electron VDFs from Helios, Cluster, and Ulysses. However, the ratio $E_{\mathrm{BP}} / T_{\mathrm{c} \|}$ corresponding to the strahl population assumes slightly lower values, between 2 (at $0.3 \mathrm{au}$ ) and 5 (at $3 \mathrm{au}$ ). Similar values are obtained by Landi et al. (2012) using a kinetic BiCoP simulation. At radial distances between 1 and $3 \mathrm{au}$, they find that $E_{\mathrm{BP}} / T_{\mathrm{c} \|}$ varies between 1 and 4 and depends mainly on the density of the modeled solar wind. In a more recent study, including Cluster data, Bakrania et al. (2020) obtain the ratio of 5.5 at 1 au as well as an anti-correlation between the strahl- $E_{B P}$ and the solar wind velocity.

The ratio $E_{\mathrm{BP}} / T_{\mathrm{c} \|}$ obtained from the PSP data shown in our work (Figure 5(d)) agrees with previous Helios results. Its median value is approximately constant, $\sim 3$, up to a radial distance of $50 R_{S}$. At greater distances, it approaches $\sim 6$.

For the majority of the samples, we find $E_{\mathrm{BP}}<E_{\mathrm{C}}$, which suggests that electrons with energies less than $E_{\mathrm{C}}$ are not limited to Maxwellian core electrons but include a small part of strahl electrons. The same is seen in the BiCoP kinetic solar wind model (Berčič et al. 2021).

We present the first observational estimates of $E_{\|}$in the solar wind (Figure 5(b)). Its strength is of order a few $\mathrm{nV} \mathrm{m}^{-1}$, and, as expected, decreases with radial distance. The radial evolution is best represented by a power-law function with an index $\alpha_{\mathrm{E}}=-1.69$. This index is very close to the power index resulting from $\mathrm{BiCoP}$ simulations, $\alpha_{\mathrm{E}, \mathrm{BCP}}=-1.55$ (Berčič et al. 2021). Following the same method as for $\Phi_{\mathrm{r}, \infty}$, the dotted curve in Figure 5(b) shows a power-law fit to the data points with a fixed index of -1.55 . For comparison, we plot $E_{\|}$from the medium-collisional BiCoP run to Figure 5(b) as a black line.

The parameters $\Phi_{\mathrm{r}, \infty}$ and $E_{\|}$are related to each other as

$$
\Phi_{\mathrm{r}, \infty}=\int_{r}^{\infty} E_{\|}(r) d r
$$

If we assume that $\Phi_{\mathrm{r}, \infty}$ and $E_{\|}$follow power laws in $r$, then the difference between the power-law indices of each of the quantities should be equal to $1\left(\alpha_{\mathrm{E}}-\alpha_{\Phi}=-1\right)$. Our results agree with this theoretical relation within the measurement uncertainty, as the difference between the fitted power-law indices is 1.02 .

\subsection{Ambipolar Contribution to the Acceleration of the Solar Wind}

The total solar wind proton potential energy $\Psi(r)$ is the sum of the repulsive electric potential energy $\mathcal{E}_{\Phi}(r)$ and the attractive gravitational potential energy $\mathcal{E}_{G}(r)$. We use the fitted curve from Figure 5(a) to calculate $\mathcal{E}_{\Phi}(r)$ from $\Phi_{\mathrm{r}, \infty}$ as

$$
\mathcal{E}_{\Phi}(r)=e \Phi_{0} r^{\alpha_{\Phi}}
$$



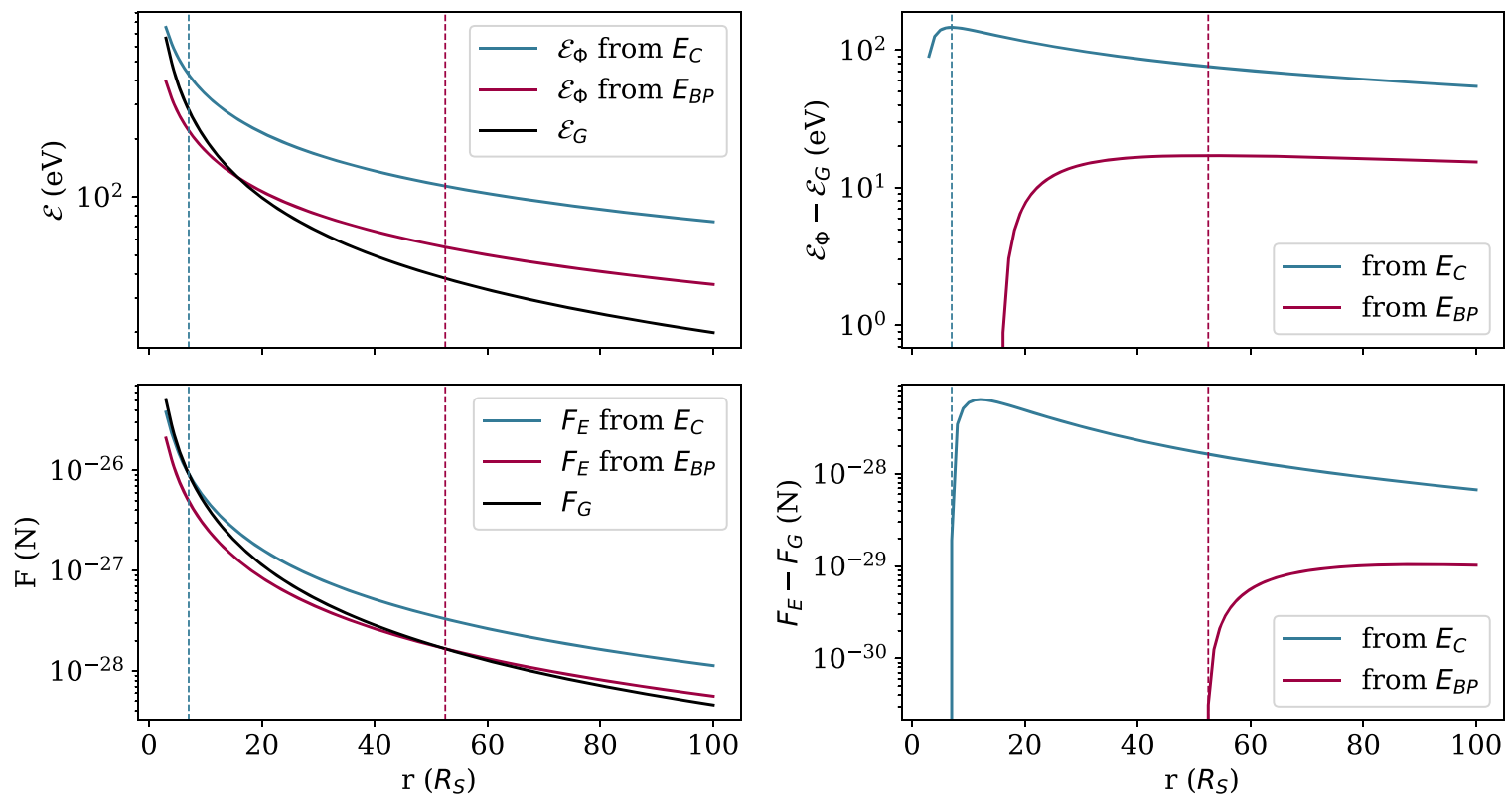

Figure 6. (a) Radial evolution of the proton gravitational energy $\left(\mathcal{E}_{G}\right)$ and the electric potential energy $\left(\mathcal{E}_{\Phi}\right)$; (b) solar wind proton energy balance; (c) radial evolution of the gravitational force $\left(F_{G}\right)$ and the electric force $\left(F_{E}\right)$ for a proton; (d) the resulting net force on a proton. In all panels, the blue color corresponds to the solution obtained from $E_{\mathrm{C}}$, and the red color to the solution obtained from $E_{\mathrm{BP}}$. Vertical dashed lines mark $r_{\max }$.

based on our determination of $E_{\mathrm{C}}$. Likewise, we use the fitted curve from Figure 5(b) to calculate $\mathcal{E}_{\Phi}(r)$ from $E_{\|}$as

$$
\mathcal{E}_{\Phi}(r)=-\frac{e E_{0}}{r_{0}\left(\alpha_{\mathrm{E}}+1\right)} r^{\alpha_{E}+1}
$$

based on our determination of $E_{\mathrm{BP}}$. The gravitational energy of a proton is defined as

$$
\mathcal{E}_{G}(r)=\frac{G M_{\mathrm{S}}}{r} m_{\mathrm{p}},
$$

where $G$ is the gravitational constant, $M_{\mathrm{S}}$ the mass of the Sun, and $m_{\mathrm{p}}$ the mass of a proton. The results are shown in the top row of Figure 6. Gravitational energy $\mathcal{E}_{G}$ decreases with radial distance faster than $\mathcal{E}_{\Phi}$, which means that $\mathcal{E}_{\Phi}$ dominates at larger radial distances, and $\Psi(r)$ peaks at a radial distance, which we denote $r_{\max }$. The kinetic theory (Scudder 1996) and BiCoP numerical simulations (Landi \& Pantellini 2003) predict a maximum of $\Psi(r)$ near the proton sonic point.

The bottom row of Figure 6 shows the radial evolution of the energy gradients, corresponding to the electric force $F_{\mathrm{E}}$ and the gravitational force $F_{\mathrm{G}}$. At small radial distances, $F_{\mathrm{G}}>F_{\mathrm{E}}$, so that the net force on protons points toward the Sun. The radial distance at which $F_{\mathrm{G}}=F_{\mathrm{E}}$ is marked with a dashed line and corresponds to the location of the total energy peak, $r_{\text {max }}$. All protons with $v_{\|} \geqslant 0$ present at $r_{\max }$ can escape the Sun's gravitational potential, as the net force on them above this distance is only positive. We calculate the radial evolution of the velocity gained by a test proton $\left(v_{\mathrm{p} \Psi}(r)\right)$ moving in the total potential $\Psi(r)$ through integration of the net force, $F(r)=F_{\mathrm{E}}(r)-F_{\mathrm{G}}(r)$, above $r_{\max }$ as

$$
v_{\mathrm{p} \Psi}(r)=\sqrt{2 \int_{r_{\max }}^{r} \frac{F(r)}{m_{\mathrm{p}}} d r} .
$$

The terminal test proton velocities $v_{\mathrm{p} \Psi}(\infty)$ result in $164 \mathrm{~km} \mathrm{~s}^{-1}$ from the method using $E_{\mathrm{C}}$, and $54 \mathrm{~km} \mathrm{~s}^{-1}$ from the method using $E_{\mathrm{BP}}$.
To calculate the bulk velocity of the protons $v_{\mathrm{p}}(r)$, we follow the exospheric approach. We assume a Maxwellian proton distribution at $r_{\max }$, with a parallel temperature $T_{\mathrm{p} \|}=0.7 \mathrm{MK}$. This is an estimation of the $T_{\mathrm{p} \|}$ at $7 R_{S}$ following from the extrapolation of the radial trends presented by Maksimovic et al. (2020). The proton parallel temperature in this simple approach does not vary with radial distance, so $v_{\mathrm{p}}(r)$ is

$$
v_{\mathrm{p}}(r)=v_{\mathrm{p} \Psi}(r)+v_{\mathrm{p}}\left(r_{\max }\right),
$$

where

$$
v_{\mathrm{p}}\left(r_{\max }\right)=\frac{2 w_{\mathrm{p} \|}}{\sqrt{\pi}} .
$$

The term $w_{\mathrm{p} \|}$ is the proton parallel thermal velocity defined as

$$
w_{\mathrm{p} \|}=\sqrt{\frac{2 k_{\mathrm{B}} T_{\mathrm{p} \|}}{m_{\mathrm{p}}}} .
$$

For simplicity we use the same $v_{\mathrm{p}}\left(r_{\max }\right)=121 \mathrm{~km} \mathrm{~s}^{-1}$ for both obtained solutions, even though they exhibit different $r_{\max }$.

Figure 7 shows the obtained velocity curves together with their asymptotic values, marked with blue and pink dashed lines. The black dashed line denotes $v_{\mathrm{p}}\left(r_{\mathrm{max}}\right)$. Proton velocity $v_{p}(r)$ resulting from $E_{\mathrm{C}}$ is greater than $v_{p}(r)$ resulting from $E_{\mathrm{BP}}$, reaching a terminal velocity of $286 \mathrm{~km} \mathrm{~s}^{-1}$. At the radial distance of $45 R_{S}$ the average observed proton velocity is $303 \mathrm{~km} \mathrm{~s}^{-1}$. The resulting $v_{p}\left(45 R_{S}\right)=233 \mathrm{~km} \mathrm{~s}^{-1}$ corresponds to $77 \%$ of the observed velocity, or $59 \%$ of the proton kinetic energy. This means that $23 \%$ of the measured solar wind velocity must be gained through other solar wind acceleration mechanisms.

Proton velocity $v_{p}(r)$ obtained from $E_{\mathrm{BP}}$ related to the SERM model is smaller, with a terminal velocity of $175 \mathrm{~km} \mathrm{~s}^{-1}$. This result at first appears unphysical, since $r_{\max }=53 R_{S}$, which would suggest that below this distance we should not observe supersonic protons at all. However, depending on the location of the solar wind acceleration by mechanisms other than $E_{\|}$, the 


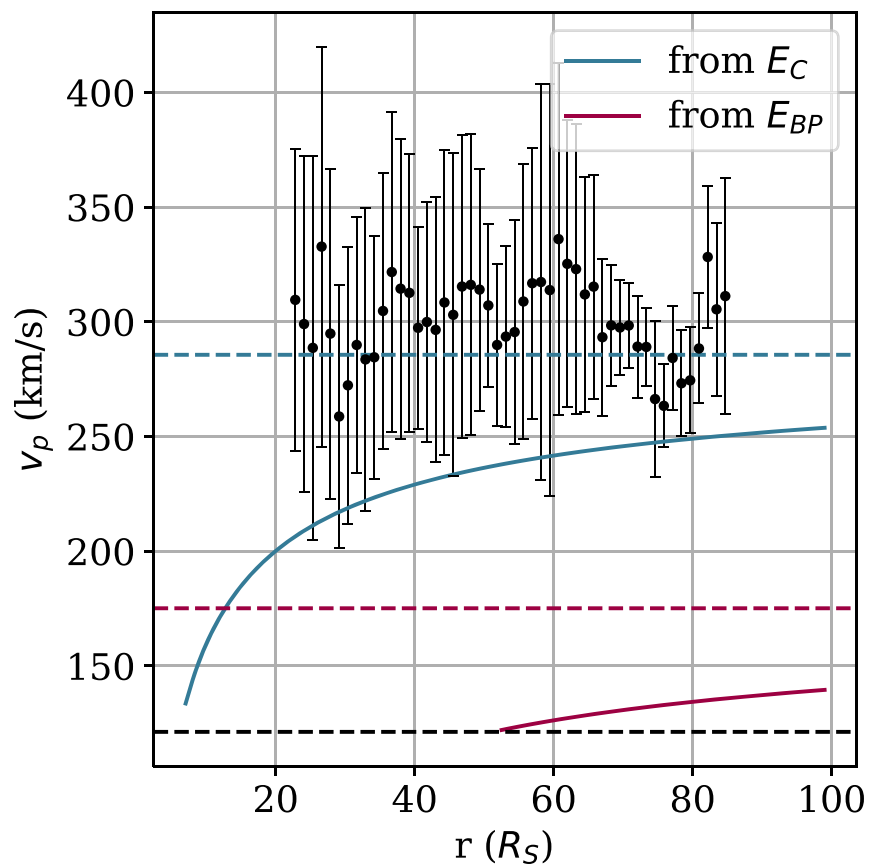

Figure 7. Radial evolution of the solar wind proton velocity $\left(v_{\mathrm{p}}(r)\right)$ obtained via Equation (18). The blue and pink lines mark the solutions resulting from different models and different features of the electron VDFs. The dashed blue and pink lines mark their asymptotic values. The black dashed line marks the bulk velocity $v_{\mathrm{p}}\left(r_{\max }\right)$ corresponding to $T_{\mathrm{p} \|}=0.7 \mathrm{MK}$. The black dots with error bars show the mean value and the standard deviation of the observed solar wind velocity binned along the radial direction in the same way as in Figure 5.

contribution of the ambipolar acceleration could increase. Additional kinetic energy close to the Sun could produce a positive net force at smaller radial distances, creating more space for the ambipolar acceleration. Comparing the obtained terminal speed with the typical solar wind speed at $1 \mathrm{au}$, we find that the $E_{\|}$is responsible for $44 \%$ of the solar wind velocity and $19 \%$ of proton kinetic energy.

Note that this is only a crude estimation, as the model we use is simplified and includes strong assumptions. That said, the statistical errors arising from the data analysis and the fits in Figures 5(a) and (b) are small. We do not include them in Figure 7, because this could be misleading for the reader.

Since this is the first effort to empirically quantify the acceleration of the solar wind by $E_{\|}$, it is difficult to make conclusions on which of the separately obtained results is more valid. Potential $\Phi_{\mathrm{r}, \infty}$ calculated from $E_{\mathrm{C}}$ is potentially overestimated, because we cannot be sure that the energy associated with the sunward deficit directly corresponds to the electron cutoff in collisionless exospheric models. The boundary could be, as a consequence of Coulomb collisions, pushed toward higher energies.

However, $E_{\|}$may be underestimated. In our analysis, we use the assumption that the separatrix between the overdamped and underdamped region is the same in the direction along with and opposite to the direction of the electric force on the electrons. We use this approximation because it appears consistent with the BiCoP simulations (Berčič et al. 2021), but theoretical work by Dreicer (1960) and Fuchs et al. (1986) suggests that the boundary is asymmetric and appears at a higher energy in the direction opposite to the electric force. This would in our analysis lead to a multiplication factor in Equation (3) and consequentially higher terminal solar wind velocities. Further investigations are needed to relate the $2 \mathrm{D}$ shape of the separatrix in the observed VDFs to the 2D shape predicted by theoretical models.

\section{Conclusions}

We analyze electron VDFs measured by PSP in the near-Sun solar wind during its orbits 4-7. We identify the electron energies at which the measured distribution departs from the biMaxwellian core electron fit in the direction parallel and antiparallel to the magnetic field. In the sunward part of phase space, we define the cutoff energy $\left(E_{\mathrm{C}}\right)$ that marks the appearance of the sunward electron deficit. In the anti-sunward portion of phase space, we define the strahl break-point energy $\left(E_{\mathrm{BP}}\right)$ that marks the start of the strahl population. While the strahl is detected in almost all of the analyzed distributions, the sunward deficit is only found in $56.8 \%$ of the cases. The relative amount of electron VDFs with a sunward deficit decreases for larger radial distances.

We relate $E_{\mathrm{C}}$ to the electron cutoff in exospheric solar wind models, which allows us to estimate the ambipolar potential between the observation point and the asymptotic potential at large heliocentric distances $\left(\Phi_{\mathrm{r}, \infty}\right)$. The resulting $\Phi_{\mathrm{r}, \infty}$ decreases with radial distance as $r^{-0.66}$. Its radial trend agrees with the results of the kinetic BiCoP model (Berčič et al. 2021), while its magnitude is slightly smaller than $\Phi_{\mathrm{r}, \infty}$ obtained numerically.

We assume that $E_{\mathrm{BP}}$ represents the separatrix between collisionally overdamped and underdamped regions of phase space, defined in the Steady Electron Runaway Model (Scudder 2019a). This allows us to estimate the ambipolar electric field in the solar wind $\left(E_{\|}\right)$. The estimated $E_{\|}$is of order $1 \mathrm{nV} \mathrm{m}^{-1}$ and decreases with radial distance as $r^{-1.69}$.

We finally calculate the total proton potential energy $\Psi(r)$ separately from $\Phi_{\mathrm{r}, \infty}$ and $E_{\|}$to estimate the contribution of the ambipolar acceleration to the total solar wind acceleration. From the approach following the exospheric models and $E_{\mathrm{C}}$ we find a terminal solar wind velocity $286 \mathrm{~km} \mathrm{~s}^{-1}$. Following the SERM model and $E_{\mathrm{BP}}$ we find a terminal solar wind velocity $175 \mathrm{~km} \mathrm{~s}^{-1}$. In the first case we are able to directly compare the observed solar wind velocity with the calculated ambipolar contribution, which amounts to $77 \%$ at the radial distance of $45 R_{S}$.

The SWEAP and FIELDS experiments on the Parker Solar Probe spacecraft were designed and developed under NASA contract NNN06AA01C. L.B., C.J.O., and D.V. are supported by STFC Consolidated Grant ST/S000240/1. D.V. is supported by STFC Ernest Rutherford Fellowship ST/ P003826/1.

\section{ORCID iDs}

Laura Berčič (iD https://orcid.org/0000-0002-6075-1813 Milan Maksimović (DD https://orcid.org/0000-0001-6172-5062 Jasper S. Halekas (iD https://orcid.org/0000-0001-5258-6128 Simone Landi (ib https://orcid.org/0000-0002-1647-6121 Christopher J. Owen (1D https://orcid.org/0000-00025982-4667

Daniel Verscharen (ib https://orcid.org/0000-0002-0497-1096 Davin Larson (i) https://orcid.org/0000-0001-5030-6030 Phyllis Whittlesey (i) https://orcid.org/0000-0002-7287-5098 Samuel T. Badman (i) https://orcid.org/0000-0002-6145-436X Stuart. D. Bale (i) https://orcid.org/0000-0002-1989-3596 Anthony W. Case (iD https://orcid.org/0000-0002-3520-4041

Keith Goetz (D) https://orcid.org/0000-0003-0420-3633 
Peter R. Harvey (1) https://orcid.org/0000-0002-6938-0166

Justin C. Kasper iㅛ https://orcid.org/0000-0002-7077-930X

Kelly E. Korreck 나 https://orcid.org/0000-0001-6095-2490

Roberto Livi (i) https://orcid.org/0000-0002-0396-0547

Robert J. MacDowall (ํ) https://orcid.org/0000-0003-

3112-4201

David M. Malaspina (1) https://orcid.org/0000-0003-

1191-1558

Marc Pulupa (1) https://orcid.org/0000-0002-1573-7457

Michael L. Stevens (1) https://orcid.org/0000-0002-7728-0085

\section{References}

Agudelo Rueda, J. A., Verscharen, D., Wicks, R. T., et al. 2021, JPIPh, 87, 905870228

Bakrania, M. R., Rae, I. J., Walsh, A. P., et al. 2020, A\&A, 639, A46

Bale, S. D., Goetz, K., Harvey, P. R., et al. 2016, SSRv, 204, 49

Bercic, L., Verscharen, D., Owen, C. J., et al. 2021, A\&A, in press

Berčič, L., Landi, S., \& Maksimović, M. 2021, JGRA, 126, e28864

Berčič, L., Larson, D., Whittlesey, P., et al. 2020, ApJ, 892, 88

Berčič, L., Maksimović, M., Landi, S., \& Matteini, L. 2019, MNRAS, 486, 3404

Bieber, J. W., Wanner, W., \& Matthaeus, W. H. 1996, JGR, 101, 2511

Boldyrev, S., Forest, C., \& Egedal, J. 2020, PNAS, 117, 9232

Boldyrev, S., \& Horaites, K. 2019, MNRAS, 489, 3412

Bourouaine, S., \& Chandran, B. D. G. 2013, ApJ, 774, 96

Case, A. W., Kasper, J. C., Stevens, M. L., et al. 2019, ApJ, 246, 43

Cattell, C., Breneman, A., Dombeck, J., et al. 2021, ApJL, 911, L29

Chandran, B. D. G., Li, B., Rogers, B. N., Quataert, E., \& Germaschewski, K. 2010, ApJ, 720, 503

Cranmer, S. 2002, in Proc. of the SOHO 11 Symp. on From Solar Min to Max: Half a Solar Cycle with SOHO, ed. A. Wilson, ESA SP-508 (Noordwijk: ESA), 361

David, C., Gabriel, A. H., Bely-Dubau, F., et al. 1998, A\&A, 336, 90

Dreicer, H. 1959, PhRv, 115, 238

Dreicer, H. 1960, PhRv, 117, 329

Dusenbery, P. B., \& Hollweg, J. V. 1981, JGR, 86, 153

Feldman, W. C., Asbridge, J. R., Bame, S. J., Montgomery, M. D., \& Gary, S. P. 1975, JGR, 80, 4181

Fox, N. J., Velli, M. C., Bale, S. D., et al. 2016, SSRv, 204, 7

Fuchs, V., Cairns, R. A., Lashmore-Davies, C. N., \& Shoucri, M. M. 1986, $\mathrm{PhFl}, 29,2931$

Halekas, J. S., Berčič, L., Whittlesey, P., et al. 2021b, ApJ, 916, 16

Halekas, J. S., Whittlesey, P., Larson, D. E., et al. 2020, ApJ, 246, 22

Halekas, J. S., Whittlesey, P. L., Larson, D. E., et al. 2021a, A\&A, 650, A15

Hollweg, J. V. 1999, JGR, 104, 24781

Horaites, K., Boldyrev, S., Wilson, L. B., Viñas, A. F., \& Merka, J. 2018, MNRAS, 474, 115

Jagarlamudi, V. K., Alexandrova, O., Berčič, L., et al. 2020, ApJ, 897, 118

Jeong, S.-Y., Verscharen, D., Wicks, R. T., \& Fazakerley, A. N. 2020, ApJ, 902,128

Jockers, K. 1970, A\&A, 6, 219
Kajdič, P., Alexandrova, O., Maksimovic, M., Lacombe, C., \& Fazakerley, A. N. 2016, ApJ, 833, 172

Karimabadi, H., Roytershteyn, V., Wan, M., et al. 2013, PhPl, 20, 012303

Kasper, J. C., Abiad, R., Austin, G., et al. 2016, SSRv, 204, 131

Kohl, J. L., Noci, G., Antonucci, E., et al. 1998, ApJL, 501, L127

Landi, S., Matteini, L., \& Pantellini, F. 2012, ApJ, 760, 143

Landi, S., \& Pantellini, F. 2001, A\&A, 372, 686

Landi, S., \& Pantellini, F. 2003, A\&A, 400, 769

Lemaire, J., \& Scherer, M. 1970, P\&SS, 18, 103

Lemaire, J., \& Scherer, M. 1971, JGR, 76, 7479

Li, X., Habbal, S. R., Hollweg, J. V., \& Esser, R. 1999, JGR, 104, 2521

Maksimovic, M., Bale, S. D., Berčič, L., et al. 2020, ApJS, 246, 62

Maksimovic, M., Pierrard, V., \& Lemaire, J. 2001, Ap\&SS, 277, 181

Maksimovic, M., Pierrard, V., \& Lemaire, J. F. 1997, A\&A, 324, 725

Maksimovic, M., Pierrard, V., \& Riley, P. 1997, GeoRL, 24, 1151

Maksimovic, M., Zouganelis, I., Chaufray, J. Y., et al. 2005, JGRA, 110 A09104

Marsch, E., \& Tu, C. Y. 2001, JGR, 106, 8357

Matthaeus, W. H., Wan, M., Servidio, S., et al. 2015, RSPTA, 373, 20140154

Mercier, C., \& Chambe, G. 2015, A\&A, 583, A101

Meyer-Vernet, N., \& Issautier, K. 1998, JGR, 103, 29705

Müller, D., Cyr, O. C., St., Zouganelis, I., et al. 2020, A\&A, 642, A1

Munro, R. H., \& Jackson, B. V. 1977, ApJ, 213, 874

Oughton, S., Matthaeus, W. H., Dmitruk, P., et al. 2001, ApJ, 551, 565

Pagel, C., Gary, S. P., de Koning, C. A., Skoug, R. M., \& Steinberg, J. T. 2007, JGR, 112, A04103

Parker, E. N. 1958, ApJ, 128, 664

Pierrard, V., Maksimovic, M., \& Lemaire, J. 1999, JGR, 104, 17021

Pierrard, V., Maksimovic, M., \& Lemaire, J. 2001, JGRA, 106, 29305

Pilipp, W. G., Miggenrieder, H., Montgomery, M. D., et al. 1987, JGR, 92, 1075

Saito, S., \& Gary, P. S. 2007, GeoRL, 34, L16109

Salem, C., Hubert, D., Lacombe, C., et al. 2003, ApJ, 585, 1147

Schwartz, S. J., \& Marsch, E. 1983, JGR, 88, 9919

Scudder, J. D. 1996, JGR, 101, 13461

Scudder, J. D. 2019a, ApJ, 885, 138

Scudder, J. D. 2019b, ApJ, 885, 148

Scudder, J. D. 2019c, ApJ, 882, 146

Scudder, J. D., \& Olbert, S. 1979, JGR, 84, 2755

Stansby, D., Berčič, L., Matteini, L., et al. 2021, A\&A, 650, L2

Štverák, Š., Maksimovic, M., Trávníček, P. M., et al. 2009, JGRA, 114, A05104

Štverák, Š., Trávníček, P., Maksimovic, M., et al. 2008, JGRA, 113, A03103

Tao, J., Wang, L., Zong, Q., et al. 2016, ApJ, 820, 22

Tu, C. Y., \& Marsch, E. 2001, JGR, 106, 8233

Verdini, A., Velli, M., Matthaeus, W. H., Oughton, S., \& Dmitruk, P. 2010, ApJL, 708, L116

Verscharen, D., Chandran, B. D. G., Jeong, S.-Y., et al. 2019, ApJ, 886, 136

Vocks, C., Salem, C., Lin, R. P., \& Mann, G. 2005, ApJ, 627, 540

Whittlesey, P. L., Larson, D. E., Kasper, J. C., et al. 2020, ApJS, 246, 74

Woodham, L. D., Horbury, T. S., Matteini, L., et al. 2021, A\&A, 650, L1

Zouganelis, I., Maksimovic, M., Meyer-Vernet, N., Lamy, H., \& Issautier, K. 2004, ApJ, 606, 542

Zouganelis, I., Meyer-Vernet, N., Landi, S., Maksimovic, M., \& Pantellini, F. 2005, ApJL, 626, L117 